\title{
sciendo
}

\section{Post-Activation Potentiation on Squat Jump Following Two Different Protocols: Traditional vs. Inertial Flywheel}

\author{
by \\ Rafael Timon ${ }^{1}$, Silvia Allemano², Marta Camacho-Cardeñosa', \\ Alba Camacho-Cardeñosa ${ }^{1}$, Ismael Martinez-Guardado ${ }^{1}$, Guillermo Olcina ${ }^{1}$
}

\begin{abstract}
Post-activation potentiation (PAP) has been defined as a major enhancement of muscular performance following a preload stimulus. The eccentric actions seem to cause a potentiating effect on subsequent explosive exercises. The aim of this study was to determine whether a protocol of squat exercise using an inertial flywheel could have a potentiating effect on jump performance. Sixteen physically active volunteers participated in the study (age: $21.8 \pm 2.7$ years; body mass index: $23.6 \pm 3$ ). All participants completed two different protocols on separate days: a Traditional Protocol (using a half squat with a guided barbell) and an Inertial Flywheel Protocol (using a half squat with an inertial flywheel). Both protocols were similar and consisted of $3 \times 6$ reps at the load that maximized power, with a 3-minute rest interval between sets. The squat jump (SJ) was measured by a contact platform at baseline, and four, eight and twelve minutes after the PAP stimulus. A two-way ANOVA with repeated measures was performed to analyze significant differences over time. There were significant increases of SJ height $(p=0.004, d=0.665)$, velocity $(p=0.003$, $d=0.688)$ and power $(p=0.004, d=0.682)$ from baseline after the inertial flywheel protocol. A significant interaction effect (time $x$ protocol) was observed, showing that the inertial flywheel protocol had a potentiating effect on the jump performance compared to the traditional protocol, more specifically at 4 and 8 minutes after the PAP stimulus. In conclusion, the inertial flywheel protocol showed a potentiating effect on the squat jump performance, thus this preconditioning activity could be useful during the warm-up before the competition.
\end{abstract}

Key words: muscle pre-activation, eccentric overload, conical pulley, half squat, explosive movement.

\section{Introduction}

Post-activation potentiation (PAP) has been defined as an acute enhancement of muscular performance following a preload stimulus with maximal or submaximal muscle actions (Gołaś et al., 2017; Hughes et al., 2016). Different physiological mechanisms have been proposed to explain the PAP. On the one hand, the phosphorylation of myosin regulatory light chains, what is related to increased rates of force development and peak isometric force (Baudry and Duchateau, 2007). On the other hand, previous muscle contraction could raise the excitability of $\alpha$-motoneurons, what may raise the fast-twitch fiber contribution (Koziris, 2012). In addition to this, some authors have even suggested a shortening of the pennation angle that may positively influence the force transmission to the tendon (Tillin and Bishop, 2009).

This mechanism of potentiation is used during the warm-up to improve competitive performance, especially for high-power movements as jumps, throws or sprints (Gołaś et al., 2016). The balance between PAP and fatigue will determine the effect on the subsequent exercise, improving muscle performance if potentiation is greater than fatigue (Andrews et

1 - Faculty of Sport Sciences. University of Extremadura. Cáceres, Spain.

2 - Scuola di Scienze Motorie. Universita Degli Studi di Milano. Milan, Italy. 
al., 2016). According to the scientific literature, PAP can be influenced by several factors, such as the type of exercise during the previous stimulus (intensity, volume, number of sets, range of motion and specificity of movement), the recovery period following the stimulus, the type of subsequent activity, and the training status of participants (Seitz and Haff, 2016; Tillin and Bishop, 2009).

However, to the best of our knowledge there is no research regarding the potentiation effect of an inertial flywheel protocol on the squat jump performance. Nonetheless, a previous study found that performance of 4 repetitions of lunge exercise on the YoYo flywheel device (YoYo Technology AB, Stockholm, Sweden) during the warm-up improved swimming start performance (Cuenca-Fernández et al., 2015). During the concentric phase of the movement, the flywheel rotates and the device stores kinematic energy. After that, the athlete must resist the inertial force of the flywheel and brake during the eccentric phase of the movement. This braking action involves an eccentric overload that causes a maximal voluntary contraction (Norrbrand et al., 2010). Eccentric contractions are characterized by higher solicitation of type II fibers and greater cortical activity (Maroto-Izquierdo et al., 2017), attaining a greater power and muscle activation compared with free weight resistance exercises (Norrbrand et al., 2010). In addition, eccentric actions activate elastic components in the muscle by the stretch-shortening cycle, increasing the production of force in subsequent actions (Hof, 2003). Several studies have concluded that the eccentric contraction prior to the explosive exercises stimulated the mechanism of PAP (Gołaś et al., 2016, 2017a; Hughes et al., 2016). On the other hand, the flywheel technology allows to individualize training by identifying the inertial load where peak power occurs for each individual, so all repetitions can be performed with maximal voluntary effort and maximal possible speed during exercise (de Hoyo et al., 2015; Martinez-Aranda and Fernandez-Gonzalo, 2016). Previous studies stated that PAP obtained with ballistic exercises was greater to that elicited with nonballistic exercises (Seitz and Haff, 2016; Suchomel et al., 2016). Likewise, a preconditioning activity based on plyometric exercises (involving activation of the stretch- shortening cycle) seems to induce a PAP effect slightly higher than moderate or high intensity concentric workouts (Seitz and Haff, 2016).

Based on this background, it was considered relevant to evaluate the potentiation effect of an inertial flywheel protocol. Thus, the purposes of this study were to determine the PAP effect of an inertial flywheel protocol on squat jump performance, and to compare the potentiation effect of this protocol with the effect caused by a traditional protocol performed with a guided barbell machine.

\section{Methods}

\section{Participants}

Sixteen physically active students ( 9 males and 7 females) volunteered for this study (age: $21.8 \pm 2.7$ years; body mass: $70.4 \pm 12.7 \mathrm{~kg}$; body height $172.2 \pm 9.8 \mathrm{~cm}$; body mass index: $23.6 \pm 3.0$; $\%$ fat mass: $20.1 \pm 7.5)$. They were identified as highly or moderately active individuals according to the International Questionnaire of Physical Activity (Craig et al., 2003). All participants were free of any musculoskeletal injuries in the lower limbs and they were able to perform several squat repetitions with heavy loads (evaluated during a practice session). The subjects attended the laboratory at the same time of the day $( \pm 2 \mathrm{~h})$, without having performed strenuous lower limbs exercises in the preceding $48 \mathrm{~h}$, and without having ingested any type of stimulating substance or alcohol in the 12 hours prior to the session. All testing and training protocols were explained and written informed consent was obtained from all participants before starting the study. The research was approved by the University Ethics Committee and met the requirements of the Declaration of Helsinki.

\section{Design and Procedures}

A randomized and counterbalanced crossover design was used to determine the PAP effect on the subsequent squat jump (SJ) of two different protocols: a Traditional Protocol (TP) vs an Inertial Flywheel Protocol (IFP). During the first week, two practice sessions separated with a minimum of 3 days were held to familiarize all participants with the training and testing protocols. Each of them focused solely on one of the training protocols. In the following two weeks, participants attended two testing sessions, which were separated by 1 week and performed 
at the same time of day. All sessions were preceded by a warm-up consisting of five minutes of cycling on an ergometer at 50 watts and 60 rpm, followed by fifteen seconds of skipping and eight free bodyweight squats.

Practice sessions. Measurements of body mass, height, body mass index and $\%$ fat mass were performed. Afterwards, the participants practiced the squat exercise that would be performed during testing. The technique of performance was assessed by researchers and instructions were given to each participant to perform the exercise properly. The familiarization movements were performed with low loads and the participants repeated the exercise until they felt confident. Furthermore, a progressive test was completed to determine the peak power $\left(\mathrm{P}_{\max }\right)$ and define the load to be used during the testing sessions. Finally, the SJ test was explained and the participants were instructed to perform the jumps at the angle required for the evaluation.

Testing sessions. After the warm-up and before performing the baseline SJ test, the skin temperature of the thigh and blood lactate concentration were measured. These control variables were recorded to ensure that both protocols were carried out under the same muscle conditions. Next, the SJ was evaluated. Two SJs were performed with a rest interval of $30 \mathrm{~s}$. The average of the two jumps was recorded as baseline value, and these data were also used to calculate the intraclass correlation coefficient (ICC) between sessions. Once this measurement was done, the protocols started.

The TP was performed in a Smith machine with a guided barbell (Shock Multipower, Telju Fitness, Spain), which reduced variability among subjects by standardizing squat technique (Figure 1). The guided barbell was centered on the shoulder and feet shoulder width apart. The TP consisted of 3 sets of 6 bilateral repetitions of the half squat (knee flexion of $90^{\circ}$ ) at $P_{\max }$ (with a workload of $68 \pm 5.7 \% 1 \mathrm{RM}$ ), and 3 min rest intervals between sets. The participants were asked to perform the protocol with a tempo of 1/0/0.5/0 (Wilk et al., 2018) (1-s eccentric phase, no break in the transition phase, a 0.5 -s concentric phase and no rest before the next repetition), trying to reach the maximal velocity in the concentric phase. The participant's feet did not leave the ground and the barbell was not allowed to leave the participant's shoulders.

The IFP was performed using an inertial flywheel device (Eccoteck training force, Byomedic Systems, Spain). This device consists of a cone that is attached above the flywheel, and a tether that is rolled or unrolled when the cone rotates, generating a momentum of inertia. The flywheel weighed $3 \mathrm{~kg}$ and an extra load in opposition could be coupled to increase the momentum of inertia. Each extra load weighed 462 grams and the distance from the rotating cone to the extra load was $11.5 \mathrm{~cm}$. The tether from the flywheel device was passed through a round pulley anchored to the ground and hooked to a hip belt of the tested participant. The participant was in an upright position, at a distance of $1.50 \mathrm{~m}$ from the device, with shoulders and hip joints in alignment, and feet shoulder-width apart (Figure 1). The training protocol consisted of 3 sets of 6 bilateral repetitions of the half squat at $P_{\max }$, and 3 min rest intervals between sets. Participants were requested to lift the load with the same tempo as in the $\mathrm{TP}$, which ranged from $90^{\circ}$ knee flexion to near full extension. During the eccentric actions, participants had to resist gently and break the movement at about $90^{\circ}$ knee flexion.

Later, 1 min after completing the protocol, the final blood lactate sample was taken. Four minutes after the stimulus, and just before performing the SJ, the skin temperature was measured again. At this time, the PAP effect was evaluated by the SJ that was performed at the $4^{\text {th }}$, $8^{\text {th }}$ and $12^{\text {th }}$ minute after the end of the activating exercise. Finally, 30 minutes after the PAP stimulus, the rated perceived exertion (RPE) was also assessed to determine the internal load of the both protocols.

\section{Measures}

Several anthropometric measurements were taken at the beginning of the first practice session to characterize the individuals. Body height was evaluated using a portable stadiometer (Seca 213, Germany). Body mass, body mass index and \% fat were assessed using a bioelectrical impedance analyzer (BF-350, Tanita Europe BV, The Netherlands). Hydration prior to body composition testing was not allowed.

$P_{\max }$ was calculated in the practice sessions to determine the load to be used during the testing sessions. The equipment and techniques employed were the same as during the 
testing sessions. In accordance with the measurement protocol proposed by de Hoyo et al. (2015), each participant had to perform an incremental protocol. The participants were positioned in a half squat position (knee flexion of $\left.90^{\circ}\right)$, both in the TP and IFP, and were instructed to extend the knees fully. For the concentric phase, the participant was instructed to move the load as quickly as possible in an attempt to maximize power output, and this movement was repeated four times. After a rest interval of 3 minutes, the load was increased by $5-10 \mathrm{~kg}$ in the $\mathrm{TP}$, or by 2 extra loads (924 g) positioned facing each other inside the flywheel in the IFP. The test finished when there was a decrease in power output as compared with the previous trial. Power output was measured by a linear transducer or a rotary axis encoder (Chronojump Encoder, Boscosystem, Spain) coupled to the guided barbell or to the flywheel device, respectively. The loads that maximized the peak power during both protocols were $74.3 \pm 15.9 \mathrm{~kg}$ (to develop $P_{\max }=1127.2 \pm 428.4$ Watts) in the Smith machine, and $7.53 \pm 0.90 \mathrm{~kg}$ (to develop $P_{\max }$ $=1007.6 \pm 291.5$ Watts) in the flywheel device.

During the testing session, all SJs were performed on a portable contact platform (Chronojump; Boscosystem, Spain) where data of jump height, velocity and power were instantaneously recorded by free software distributed by the manufacturer (Software chronojump V1.8.0, Boscosystem, Spain). The participants started from a stationary squat position $\left(90^{\circ}\right)$, with hands on the hips, before jumping upwards. On the command, the participant extended their knees and jumped as high as possible while maintaining the hands on the waist and landed with both feet. Baseline measurement was the average value of two squat jumps performed after the warm-up and separated by $30 \mathrm{~s}$. Subsequently, after the PAP stimulus, another SJ was performed and recorded at the $4^{\text {th }}, 8^{\text {th }}$ and $12^{\text {th }}$ minute.

Skin temperature was measured from the thigh of the right leg by an infrared thermometer (TAC 5000, Exergen, USA) to check that the temperature was the same under both conditions. Temperature was taken before the baseline SJ and just before the SJ performed $4 \mathrm{~min}$ after the PAP stimulus.

Capillary blood lactate and the RPE were analyzed to determine the internal load during the testing sessions and verify whether the results obtained were not due to possible differences in the fatigue level induced by both protocols. Samples were drawn from the fingertip of participants before the baseline SJ and 1 min after the PAP stimulus. Lactate concentration was measured with a fast and reliable portable analyzer (Lactate Scout+, SensLab $\mathrm{GmbH}$, Germany) that uses an enzymatic-amperometric detection method and requires only $0.5 \mu \mathrm{L}$ of blood. The RPE was assessed by the OMNIResistance Exercise Scale (OMNI-RES) which is a validated RPE for resistance exercise (Robertson et al., 2003). The OMNI-RES consisted of 10 reporting options between 1 (extremely easy) and 10 (extremely hard). Thirty minutes after finishing the protocols, a copy of this scale was given to the participants and they rated how hard the whole training session had been.

\section{Statistical analysis}

Statistical analyses were performed using IBM SPSS Statistics for Windows, version 23.0. (IBM Corp., Armonk, New York, USA). The Shapiro-Wilk test was applied to verify a normal distribution of data and the Levene's test was used to assess the homogeneity of variance. A two-way analysis of variance with repeated measures and the Bonferroni post hoc test were conducted to evaluate the potentiation effect on dependent variables (SJ height, velocity and power). The factors were protocols (traditional and inertial flywheel) and time with 4 levels (baseline, $4 \mathrm{~min}, 8 \mathrm{~min}$ and $12 \mathrm{~min}$ ). The time $\mathrm{x}$ protocol interaction effect was tested using the multivariate criterion of Pillai's trace. Reliability was determined according to the ICC with 95\% confidence intervals between the two testing sessions. The ICC was calculated between the baseline values obtained in the squat performed in the inertial flywheel session and the squat performed in the traditional session. Moreover, standard error of measurement (SEM) and smallest real difference (SRD) were assessed to detect the real variation in the dependent variables (Beckerman et al., 2001). In order to facilitate the interpretation and comparison of results with other studies, SEM\% ([SEM/mean values of 2 testing sessions] $x$ 100) and SRD\% ([SRD/ mean values of 2 testing sessions) $x$ 100] were shown. The effect size (ES) was calculated 
for all dependent variables over time using the Cohen's d. The magnitude of effect was classified as trivial (0.25), small (between 0.25 and 0.50), moderate (between 0.50 and 1.0) and large (>1.0). On the other hand, a one way ANOVA was performed to analyze differences of skin temperature, blood lactate concentration and RPE values between protocols. The significance level was set at $p \leq 0.05$, with a confidence level of $95 \%$. Means and standard deviations (SD) were used as descriptive statistics.

\section{Results}

Table 1 shows the potentiation effects on the SJ after both protocols. There were significant increases of SJ height $(p=0.004, \mathrm{ES}=0.665)$, velocity $(p=0.003, \mathrm{ES}=0.688)$ and power $(p=$ 0.004 , ES $=0.682$ ) from baseline after the IFP. Effect size for the improvement in all dependent variables was moderate (greater than 0.60). Post hoc analyses revealed that the IFP significantly improved the performance of the SJ in comparison to baseline at the $4^{\text {th }}$ and $8^{\text {th }}$ minute after completing the protocol. No significant difference was observed after the traditional protocol in any variable.

Table 2 shows the ICC values and measurement errors between testing sessions. All dependent variables had an ICC greater than 0.95 . The values of SEM\% ranged from 2.2 to $2.6 \%$ and the values of SRD\% ranged from 6.2 to $7.2 \%$. These data indicated that the measurements taken could be considered reliable.

A significant interaction effect (time $x$ protocol) was observed (Figure 2), showing that the IFP had a potentiation effect on performance (SJ height, velocity and power) compared to the TP, specifically at the $4^{\text {th }}$ and $8^{\text {th }}$ minute after the PAP stimulus.

No significant difference was observed between protocols in the variables used to monitor the internal load such as skin temperature, blood lactate concentration and the RPE (Table 3).

Table 1

Potentiation effects on squat jump performance after both protocols (Mean \pm SD)

\begin{tabular}{|c|c|c|c|c|c|c|c|}
\hline Protocols & Variables & Baseline & SJ $4^{\prime}$ & SJ $8^{\prime}$ & SJ $12^{\prime}$ & $p$ & d \\
\hline \multirow[b]{2}{*}{$\begin{array}{l}\text { Traditional } \\
\text { protocol }\end{array}$} & $\begin{array}{l}\text { Height } \\
(\mathrm{cm})\end{array}$ & $27.83 \pm 5.71$ & $27.83 \pm 5.51$ & $27.38 \pm 5.68$ & $27.10 \pm 6.15$ & 0.614 & 0.135 \\
\hline & $\begin{array}{c}\text { Velocity } \\
(\mathrm{m} / \mathrm{s})\end{array}$ & $2.32 \pm 0.25$ & $2.32 \pm 0.24$ & $2.30 \pm 0.24$ & $2.29 \pm 0.26$ & 0.557 & 0.153 \\
\hline \multirow{4}{*}{$\begin{array}{l}\text { Inertial } \\
\text { flywheel } \\
\text { protocol }\end{array}$} & Power (W) & $807.94 \pm 182.67$ & $806.31 \pm 172.51$ & $801.29 \pm 179.90$ & $797.06 \pm 183.51$ & 0.638 & 0.127 \\
\hline & $\begin{array}{l}\text { Height } \\
(\mathrm{cm})\end{array}$ & $26.97 \pm 5.26$ & $28.82 \pm 5.77 *$ & $28.87 \pm 5.94$ * & $26.72 \pm 6.17$ & 0.004 & 0.665 \\
\hline & $\begin{array}{l}\text { Velocity } \\
(\mathrm{m} / \mathrm{s})\end{array}$ & $2.25 \pm 0.23$ & $2.34 \pm 0.25$ * & $2.34 \pm 0.27^{*}$ & $2.27 \pm 0.27$ & 0.003 & 0.688 \\
\hline & Power (W) & $782.55 \pm 173.15$ & $818.40 \pm 192.54^{*}$ & $816.71 \pm 184.99^{*}$ & $791.80 \pm 185.84$ & 0.004 & 0.682 \\
\hline
\end{tabular}

*Significant differences from baseline;

SJ: Squat Jump; p: Significance level; d: Effect size (Cohen's d) 


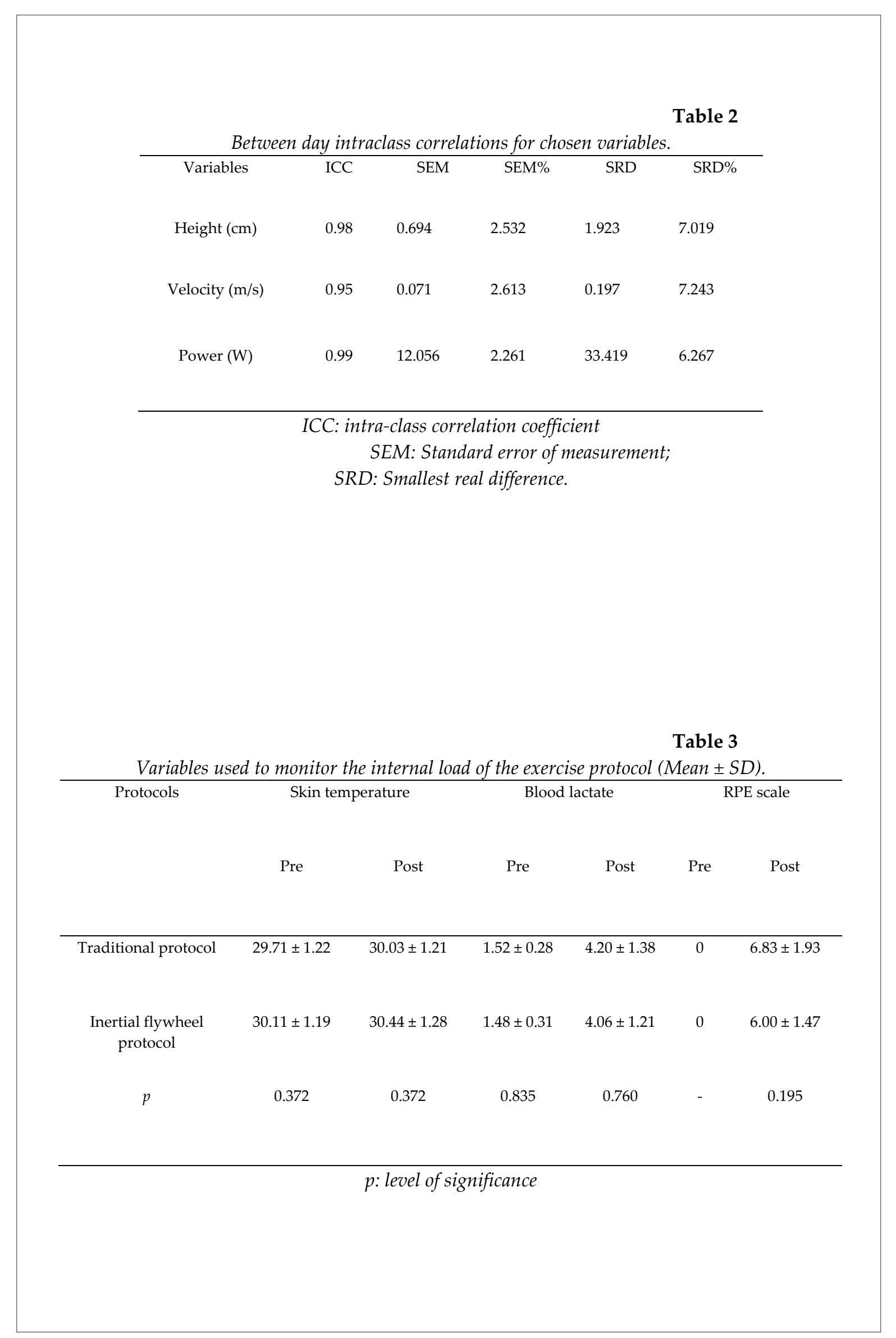




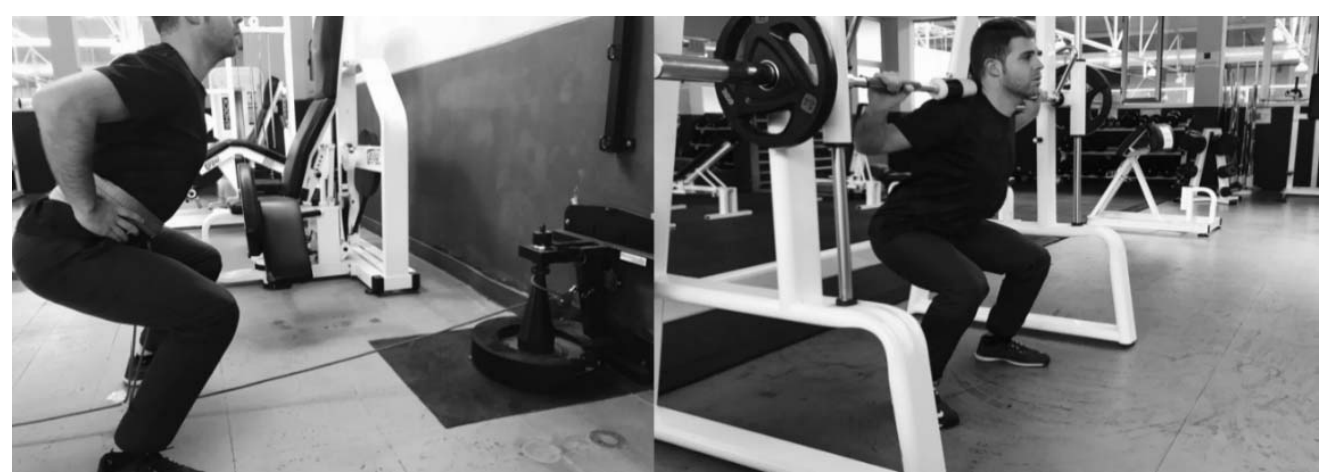

Figure 1

Half squat techniques performed in both protocols.
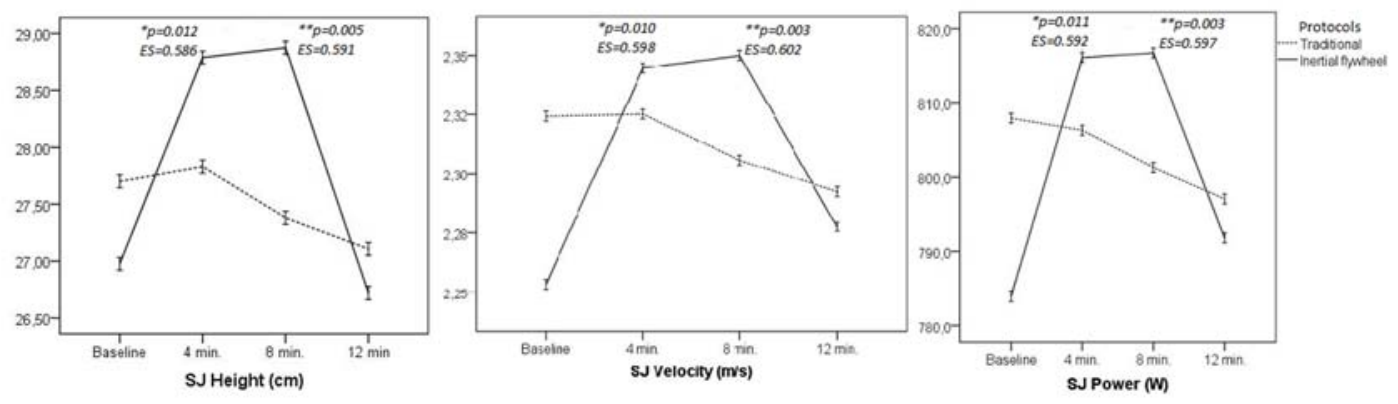

Figure 2

Time $x$ Protocol interaction effects on SJ performance over time.

\section{Discussion}

This study is the first research to observe the PAP effect of an inertial flywheel protocol on SJ performance. The results showed that the SJ significantly increased after the IFP, whereas the TP did not induce any improvement.

The rotational inertia during flywheel exercise causes a greater eccentric overload than that produced by the traditional resistance exercise (Maroto-Izquierdo et al., 2017). The type II muscle fibers are highly recruited during eccentric contractions, a very relevant fact since the PAP effect has been observed especially in this type of muscle fibers (Rixon et al., 2007). Consequently, the eccentric overload could 
explain the potentiation effect observed after our flywheel protocol. Indeed, Gołaś et al. (2016) showed that eccentric supramaximal intensities $(130 \% 1 \mathrm{RM})$ could be effective in eliciting PAP in strength trained individuals. Similar results to those shown in our study were observed in swimmers who improved power output after using a YoYo flywheel device in a previous warm up (Cuenca-Fernández et al., 2015). However, some research has concluded that the use of augmented eccentric loads prior to a 30\% 1RM squat jump failed to acutely enhance force, velocity and power (Moore et al., 2007). An explanation for this conflicting result may be that the volume and intensity of the preconditioning exercise performed during the aforementioned study (only 2 sets of 1 RM with eccentric loads of 20,50 , and $80 \%$ of the back squat $1 \mathrm{RM}$ ) were not sufficient to cause a potentiation effect.

Other factors could also explain the PAP effect on jump performance after using the flywheel device. On the one hand, the eccentricconcentric transition during flywheel training activates a powerful stretch-shortening cycle (Martinez-Aranda and Fernandez-Gonzalo, 2016). This stretch reflex could potentiate the force peak of a subsequent explosive action due to the use of stored elastic energy (Wilson and Flanagan, 2008). Previous studies have shown an acute improvement in vertical jump performance after a warm-up with specific jumping exercises, in which the stretch-shortening cycle played an important role (Chen et al., 2017). In addition, every subject in our study performed the IFP with an individual training load (inertia), that allowed to reach peak power and maximal possible velocity during the movement. The high movement velocity may have a positive PAP effect on performance and power, increasing the neuromuscular activation (de Hoyo et al., 2015). Suchomel et al. (2016) concluded that the potentiation effect on jump performance was greater when half-squat exercise was performed with maximal velocity compared to without maximal velocity. On the other hand, the relative contribution of the joints to the movement is different when comparing exercises performed with traditional weights or with an inertial flywheel. The squat exercise performed with flywheel resistance had greater impulse requirement of the hip and ankle compared to free weight (Chiu and Salem, 2006). This fact could indicate that during our IFP there was a pre-activation of a greater number of muscle fibers than when the TP was performed, which favored a better potentiation effect on the SJ.

Regarding the PAP effect after the TP, no improvement was observed in the subsequent SJ. Previous studies have concluded that the fatigue level after the preconditioning activity can increase and reduce the ability to cause PAP (Lowery et al., 2012). However, it is important to note that there were no significant differences in blood lactate concentration and the RPE when comparing both protocols. Therefore, the level of fatigue would not be the variable that explains the lack of potentiation effect after the TP. The lack of improvement in the SJ could also be influenced by the muscle temperature. A decrease in muscle temperature has been associated with a fall in muscle power (Fletcher, 2013). However, we observed in our study that the temperature in both protocols was similar. Consequently, we should seek the explanation of this lack of improvement after the TP in other factors, such as the volume, intensity or type of conditioning exercise. Hanson et al. (2007) also did not find a PAP effect on the countermovement jump (CMJ) after performing squat training with light loads (8 repetitions at $40 \%$ of $1 \mathrm{RM}$ ) and high velocity. On the contrary, several studies have reported increases in jump performance after a heavy-squat protocol lifting 2-5 RM (Chen et al., 2017; Fukutani et al., 2014), which contrasts with the findings of our study. These conflicting results could be explained by the fact that the TP of the present study had lower intensity and higher volume of repetitions.

On the other hand, the SJ improved at the $4^{\text {th }}$ and $8^{\text {th }}$ minute after the IFP, while this potentiation effect disappeared at the $12^{\text {th }}$ minute. Previous reviews have showed that the greatest PAP effect is realized after longer recovery intervals (4-8 $\mathrm{min}$ ) than after a shorter rest interval (2-3 $\mathrm{min})$, although inter-individual variability was observed (Seitz and Haff, 2016; Wilson et al., 2013). These results are similar to those found by Lowery et al. (2012), who observed that the squat jump height increased and peaked from the $4^{\text {th }}$ to $8^{\text {th }}$ min and returned to baseline by the $12^{\text {th }}$ min after performing a back squat exercise at $93 \% 1 \mathrm{RM}$. During the first 4 
minutes, the fatigue can dominate and reduce the performance, but later the fatigue would dissipate and the potentiation appears. In this sense, untrained individuals take longer to recover (about 8 minutes) and to achieve the maximum PAP responses (Seitz et al., 2014), as it happened in our study. In addition, afferent excitability caused by the muscle contraction is reported to persist for 3-10 min post exercise (Trimble and Harp, 1998), which is consistent with the time course of potentiation in our study that returned to baseline values by the $12^{\text {th }} \mathrm{min}$.

The present study had some limitations, such as the lack of data for other blood variables so as to be able to evaluate muscular fatigue and neuromuscular excitability. Moreover, the squat protocols were performed with a single load on a specific range of movement, thus further research is required to corroborate the effectiveness of the inertial flywheel to potentiate the SJ.

In conclusion, the IFP showed a potentiation effect on the SJ between the $4^{\text {th }}$ and $8^{\text {th }}$ minute after the exercise. However, the TP with a load that maximized power did not have any potentiating effect on performance of the SJ. These findings are of practical relevance for coaches and athletes who could use the inertial flywheel device to potentiate squat jump performance. This pre-conditioning strategy could be useful during the warm-up before competition, specifically in sport disciplines including explosives actions. The time interval between the $4^{\text {th }}$ and $8^{\text {th }}$ min after the preconditioning activity provides the greatest potentiation effect, thus the coaches should properly organize warming activities taking into account this time lag. Anyway, given that the PAP phenomenon depends on many variables, it remains important to individualize the conditioning protocol to the greatest possible extent.

\section{Acknowledgements}

This work was supported by the Regional Government of Extremadura (Spain) under Grant [number CTS036 GR18003].

\section{References}

Andrews SK, Horodyski JM, MacLeod DA, Whitten J, Behm DG. The Interaction of Fatigue and Potentiation Following an Acute Bout of Unilateral Squats. J Sports Sci Med, 2016; 15(4): 625-632

Baudry S, Duchateau J. Postactivation potentiation in a human muscle: effect on the rate of torque development of tetanic and voluntary isometric contractions. J Appl Physiol (1985), 2007; 102(4): 13941401. doi:10.1152/japplphysiol.01254.2006

Beckerman H, Roebroeck ME, Lankhorst GJ, Becher JG, Bezemer PD, Verbeek AL. Smallest real difference, a link between reproducibility and responsiveness. Qual Life Res, 2001; 10(7): 571-578

Chen ZR, Lo SL, Wang MH, Yu CF, Peng HT. Can Different Complex Training Improve the Individual Phenomenon of Post-Activation Potentiation? J Hum Kinet, 2017; 56: 167-175. doi:10.1515/hukin-20170034

Chiu LZ, Salem GJ. Comparison of joint kinetics during free weight and flywheel resistance exercise. J Strength Cond Res, 2006; 20(3): 555-562. doi:10.1519/R-18245.1

Craig CL, Marshall AL, Sjöström M, Bauman AE, Booth ML, Ainsworth BE, Pratt M, Ekelund U, Yngve A, Sallis JF, Oja P. International physical activity questionnaire: 12-country reliability and validity. Med Sci Sports Exerc, 2003; 35(8): 1381-1395. doi:10.1249/01.MSS.0000078924.61453.FB

Cuenca-Fernández F, López-Contreras G, Arellano R. Effect on swimming start performance of two types of activation protocols: lunge and YoYo squat. J Strength Cond Res, 2015; 29(3): 647-655. doi:10.1519/JSC.0000000000000696 
de Hoyo M, Sañudo B, Carrasco L, Domínguez-Cobo S, Mateo-Cortes J, Cadenas-Sánchez MM, Nimphius S. Effects of Traditional Versus Horizontal Inertial Flywheel Power Training on Common Sport-Related Tasks. J Hum Kinet, 2015; 47: 155-167. doi:10.1515/hukin-2015-0071

Fletcher IM. An investigation into the effect of a pre-performance strategy on jump performance. J Strength Cond Res, 2013; 27(1): 107-115. doi:10.1519/JSC.0b013e3182517ffb

Fukutani A, Takei S, Hirata K, Miyamoto N, Kanehisa H, Kawakami Y. Influence of the intensity of squat exercises on the subsequent jump performance. J Strength Cond Res, 2014; 28(8): 2236-2243. doi:10.1519/JSC.0000000000000409

Gołaś A, Maszczyk A, Zajac A, Mikołajec K, Stastny P. Optimizing post activation potentiation for explosive activities in competitive sports. J Hum Kinet, 2016; 52: 95-106. doi:10.1515/hukin-2015-0197

Gołaś A, Wilk M, Statsny P, Maszczyk A, Pajerska K, Zajac A. Optimizing Half Squat Post Activation Potential Load In Squat Jump Training For Eliciting Relative Maximal Power In Ski Jumpers. J Strength Cond Res, 2017; doi:10.1519/JSC.0000000000001917

Gołaś A, Maszczyk A, Pietraszewski P, Stastny P, Tufano JJ, Zając A. Effects of Pre-exhaustion on the Patterns of Muscular Activity in the Flat Bench Press. J Strength Cond Res., 2017a; 31(7): 1919-1924 doi: 10.1519/JSC.0000000000001755

Hanson ED, Leigh S, Mynark RG. Acute effects of heavy- and light-load squat exercise on the kinetic measures of vertical jumping. J Strength Cond Res, 2007; 21(4): 1012-1017. doi:10.1519/R-20716.1

Hof AL. Muscle mechanics and neuromuscular control. J Biomech, 2003; 36(7): 1031-1038

Hughes JD, Massiah RG, Clarke RD. The potentiating effect of an accentuated eccentric load on countermovement jump performance. J Strength Cond Res, 2016; 30(12): 3450-3455. doi:10.1519/jsc.0000000000001455

Koziris L. Postactivation Potentiation: Sometimes More Fatigue Than Potentiation. Strength Cond J, 2012; 34(6): 75-76. doi:10.1519/SSC.0b013e31826ddc07

Lowery RP, Duncan NM, Loenneke JP, Sikorski EM, Naimo MA, Brown LE, Wilson FG, Wilson JM. The effects of potentiating stimuli intensity under varying rest periods on vertical jump performance and power. J Strength Cond Res, 2012; 26(12): 3320-3325. doi:10.1519/JSC.0b013e318270fc56

Maroto-Izquierdo S, García-López D, Fernandez-Gonzalo R, Moreira OC, González-Gallego J, de Paz JA. Skeletal muscle functional and structural adaptations after eccentric overload flywheel resistance training: a systematic review and meta-analysis. J Sci Med Sport, 2017; 20(10): 943-951. doi:10.1016/j.jsams.2017.03.004

Martinez-Aranda LM, Fernandez-Gonzalo R. Effects of inertial setting on power, force, work and eccentric overload during flywheel resistance exercise in women and men. J Strength Cond Res, 2016; 31(6): 16531661. doi: 10.1519/JSC.0000000000001635

Moore CA, Weiss LW, Schilling BK, Fry AC, Li Y. Acute effects of augmented eccentric loading on jump squat performance. J Strength Cond Res, 2007; 21(2): 372-377. doi:10.1519/R-18605.1

Norrbrand L, Pozzo M, Tesch PA. Flywheel resistance training calls for greater eccentric muscle activation than weight training. Eur J Appl Physiol, 2010; 110(5): 997-1005. doi:10.1007/s00421-010-1575-7

Rixon KP, Lamont HS, Bemben MG. Influence of type of muscle contraction, gender, and lifting experience on postactivation potentiation performance. J Strength Cond Res, 2007; 21(2): 500-505. doi:10.1519/R18855.1

Robertson RJ, Goss FL, Rutkowski J, Lenz B, Dixon C, Timmer J, Frazee K, Dube J, Andreacci J. Concurrent validation of the OMNI perceived exertion scale for resistance exercise. Med Sci Sports Exerc, 2003; 35(2): 333-341. doi:10.1249/01.MSS.0000048831.15016.2A

Seitz LB, de Villarreal ES, Haff GG. The temporal profile of postactivation potentiation is related to strength level. J Strength Cond Res, 2014; 28(3): 706-715. doi:10.1519/JSC.0b013e3182a73ea3 
Seitz LB, Haff GG. Factors Modulating Post-Activation Potentiation of Jump, Sprint, Throw, and UpperBody Ballistic Performances: A Systematic Review with Meta-Analysis. Sports Med, 2016; 46(2): 231240. doi:10.1007/s40279-015-0415-7

Suchomel TJ, Sato K, DeWeese BH, Ebben WP, Stone MH. Potentiation effects of half-squats performed in a ballistic or nonballistic manner. J Strength Cond Res, 2016; 30(6): 1652-1660

Tillin NA, Bishop D. Factors modulating post-activation potentiation and its effect on performance of subsequent explosive activities. Sports Med, 2009; 39(2): 147-166

Trimble MH, Harp SS. Postexercise potentiation of the H-reflex in humans. Med Sci Sports Exerc, 1998; 30(6): 933-941

Wilk M, Golas A, Stastny P, Nawrocka M, Krzysztofik M, Zajac A. Does tempo of resistance exercise impact training volume? J Hum Kinet, 2018; 62: 241-250. doi: 10.2478/hukin-2018-0034.

Wilson JM, Duncan NM, Marin PJ, Brown LE, Loenneke JP, Wilson SM, Jo E, Lowery RP, Ugrinowitsch C. Meta-analysis of postactivation potentiation and power: effects of conditioning activity, volume, gender, rest periods, and training status. J Strength Cond Res, 2013; 27(3): 854-859. doi:10.1519/JSC.0b013e31825c2bdb

Wilson JM, Flanagan EP. The role of elastic energy in activities with high force and power requirements: a brief review. J Strength Cond Res, 2008; 22(5): 1705-1715. doi:10.1519/JSC.0b013e31817ae4a7

\section{Corresponding author:}

\section{Rafael Timon}

Sport Sciences Faculty. Universidad de Extremadura.

Av de la Universidad $\mathrm{s} / \mathrm{n}$

(10003) Cáceres

SPAIN

Phone number: 0034927257460

Fax number: 0034927257461

E-mail: rtimon@unex.es 